\title{
Effects of High-Potency Cannabis on Psychomotor Performance in Frequent Cannabis Users
}

Hollis C. Karoly, 1,* Michael A. Milburn, ${ }^{2}$ Ashley Brooks-Russell, ${ }^{3}$ Mary Brown, ${ }^{4,5}$ Jessica Streufert, Angela D. Bryan, ${ }^{1,6}$ Nicholas P. Lovrich, ${ }^{7}$ William DeJong, ${ }^{8}$ and L. Cinnamon Bidwell ${ }^{1,6}$

\begin{abstract}
Background: Recently increased access to cannabis products in the United States has been associated with increased rates of driving after cannabis use. Although numerous studies indicate that cannabis impairs psychomotor and neurocognitive functions that can affect driving ability, the determination of cannabis-impaired driving risk is complicated by the extent to which frequent cannabis users develop tolerance to THC's subjective, cognitive, and psychomotor effects, and by the fact that there is no validated behavioral or biological marker of recent cannabis use or cannabis-related impairment. This study examined the psychomotor impairment-related effects experienced by frequent cannabis users in Colorado after naturalistic consumption of smoked cannabis, both immediately and $1 \mathrm{~h}$ postuse. Results were then validated in a smaller replication sample from Washington state. Methods: In the primary Colorado study, participants $(n=70)$ used the DRUID ${ }^{\circledR}$ mobile app, a brief measure of psychomotor and cognitive domains that are sensitive to the effects of cannabis. First, participants used DRUID to establish a sober baseline impairment score. During a second appointment, they used DRUID at three time points: preuse, immediately after acutely using cannabis, and $1 \mathrm{~h}$ postuse. In the Washington replication sample, participants $(n=39)$ used DRUID before acute cannabis consumption and then every half hour for $2.5 \mathrm{~h}$.

Results: In both studies, peak DRUID impairment effects were seen immediately after cannabis use, with recovery of performance at $1 \mathrm{~h}$ postuse. Specifically, significant quadratic effects of time emerged for both studies (Colorado study: $(\beta=-0.935, S E=0.204, p<0.001$ ); Washington study: $\beta=3.0299, S E=1.3085, p<0.01$ ) Domain-specific effects were tested in the larger Colorado study and were observed for reaction time within a complex divided attention task and a postural-stability balance task.

Conclusions: These findings demonstrate that psychomotor impairment emerges immediately after acute cannabis use even in regular users, but decreases significantly $1 \mathrm{~h}$ postuse. These results underscore the potential utility of the DRUID app for assessing acute cannabis-related psychomotor impairment. Further research is needed to explore whether the DRUID app and/or the specific psychomotor functions it assesses might serve as a tool for measuring cannabis-related driving impairment. Clinical trials registration number for the Colorado Study: NCT03522103.
\end{abstract}

Keywords: cannabis; psychomotor; neurocognitive; impairment; driving

\section{Introduction}

As of January, 2020, 33 U.S. states, 4 U.S. territories, and the District of Columbia have legalized medical cannabis, whereas 11 states, 2 territories, and Washington D.C. have legalized recreational cannabis.
Adult cannabis consumption has been increasing, particularly in jurisdictions where cannabis has been legalized, ${ }^{1}$ which has led to increased rates of driving after cannabis use. According to the National Roadside Survey, the percentage of drivers testing

\footnotetext{
${ }^{1}$ Institute for Cognitive Science, University of Colorado, Boulder, Colorado, USA.

${ }^{2}$ Impairment Science, Inc., Cambridge, Massachusetts, USA.

${ }^{3}$ Department of Community and Behavioral Health, Colorado School of Public Health, University of Colorado Anschutz Medical Campus, Aurora, Colorado, USA.

${ }^{4}$ Advanced Integrative Medical Science Institute, Seattle, Washington, USA.

${ }^{5} \mathrm{SMJ}$ Consulting, Seattle, Washington, USA.

${ }^{6}$ Department of Psychology and Neuroscience, University of Colorado, Boulder, Colorado, USA.

${ }^{7}$ Department of Criminal Justice and Criminology, Washington State University, Pullman, Washington, USA.

${ }^{8}$ Department of Public Health and Community Medicine, Tufts University School of Medicine, Boston, Massachusetts, USA.

*Address correspondence to: Hollis C. Karoly, PhD, Institute of Cognitive Science, University of Colorado, 1777 Exposition Drive, Boulder, CO 80309, USA, E-mail: hollis.karoly@colorado.edu
} 
positive for cannabis increased from $8.6 \%$ in 2007 to $13.0 \%$ in $2013-2014 .^{2}$

Detecting whether drivers suspected of driving under the influence are experiencing cannabis-associated impairment is critical for protecting public safety, but presently there is no reliable tool for doing so. Both the Standard Field Sobriety Test ${ }^{3,4}$ and Drug Recognition Expert evaluations ${ }^{5}$ have limited efficacy for assessing cannabis impairment, ${ }^{6}$ and there are no biochemical (e.g., blood, saliva) THC tests that can reliably identify either recent use or impairment. ${ }^{7}$ From a prevention standpoint, cannabis users themselves could benefit from having a tool to assess their impairment level before they get behind the wheel.

Research shows that cannabis use negatively impacts complex psychomotor performance and related driving ability. For both occasional and frequent users, cannabis decreases both psychomotor and neurocognitive performance, especially on divided-attention (performance on a task when presented with a distraction) and error compensation (noticing and correcting mistakes during a task) tasks. ${ }^{8}$ Likewise, driving simulator studies find that acute cannabis use increases lane weaving and decreases performance on critical tracking and reaction time tasks. ${ }^{9,10}$

Some decrements in psychomotor performance, particularly divided attention and postural sway (horizontal movement around a person's center of gravity), persist even when cannabis users are not acutely intoxicated. ${ }^{11,12}$ Other studies show that frequent users have persisting negative effects on impulsivity ${ }^{13,14}$ and executive function. ${ }^{15}$ Human neuroimaging research confirms that cannabis users experience deficits in brain activation in motor control regions that persist beyond acute intoxication. ${ }^{16}$ The varied psychomotor decrements associated with cannabis use acutely (as well as the finding that some degree of impairment may persist beyond acute intoxication) make it challenging to determine cannabisrelated impairment.

Another factor complicating our ability to determine cannabis impairment is the availability and popularity of cannabis concentrate products that have largely not been examined in research studies. ${ }^{17-20}$ Concentrates typically contain significantly higher percentage THC compared with flower cannabis, with cannabis concentrates up to $90 \%$ THC now commonly used and available on the legal market. ${ }^{21}$ The effects of these high-potency products on subjective intoxication and cognitive and motor impairment are for the most part unknown. In our group's recent study among individuals who were frequent users of legal market flower and concentrated cannabis, we demonstrated that these participants showed tolerance such that acute cannabis use did not impact many cognitive tasks (e.g., inhibitory control and working memory). However, balance performance emerged as one psychomotor function that is impaired after acute use but appears to recover within $1 \mathrm{~h}^{22}$ These findings are among the only data on the acute cognitive and psychomotor effects of legal market forms of cannabis among regular users and need to be explored further using additional tasks and in other populations. Taken together, the existing literature underscores the importance of gaining a better understanding of the extent and time course of the effects of various cannabis preparations and THC concentrations on psychomotor tasks, balance, and cognition, all of which may adversely affect driving ability.

The purpose of this study is to explore the utility of a brief mobile instrument, the DRUID ${ }^{\circledR}$ mobile application (app), which measures psychomotor and cognitive domains that have previously been shown to be sensitive to drug effects and may also be relevant to driving. ${ }^{23,24}$ We used a naturalistic design wherein individuals were tested on the DRUID before and after ad libitum use of legal market cannabis flower and concentrate products. We hypothesize that impairment on the DRUID battery would increase after acute cannabis use, and decrease from the immediate postuse to $1 \mathrm{~h}$ postuse time point. We tested this hypothesis with two groups of participants located in states with legal market access to cannabis. The primary sample included participants from Colorado who were participating in a larger study of the effects of naturalistic cannabis administration on subjective, cognitive, and psychomotor effects. ${ }^{22}$ We then sought to validate these results through testing the same hypotheses using a smaller replication sample from Washington state.

\section{Materials and Methods}

\section{Colorado study}

The data for this study are from a larger investigation of the impact of naturalistic cannabis administration on established measures of subjective, cognitive, and psychomotor effects. $^{22}$

Participants. Participants were regular users of either flower cannabis or concentrates, recruited from Boulder and Denver using posted advertising, social media postings, and flyers. Research staff screened participants through telephone. Inclusion criteria were (1) age 2170 years, (2) used cannabis at least four times in the 
past month, (3) had experience with the highest concentration cannabis used in the study (flower cannabis: 24\% THC; concentrate: $90 \%$ THC), (4) had not used nonprescription drugs in past 60 days (verification by urine drug screen), (5) not using tobacco daily, (6) used alcohol $\leq 2$ times per week (with $\leq 3$ drinks per occasion), (7) not pregnant or trying to become pregnant, and (8) not receiving treatment for psychosis, bipolar disorder, or schizophrenia. All exclusion criteria were determined by the larger study, which has been described previously. ${ }^{22}$ The aims of this study involve examining the acute effects of cannabis on subjective mood, craving, and intoxication. The conservative alcohol consumption criteria and exclusion for daily tobacco use were determined to ensure that subjects were not experiencing alcohol or tobacco withdrawal or craving during the experimental session, which could impact their subjective experiences during acute cannabis administration.

Procedure. The research protocol was approved by the University of Colorado-Boulder IRB, as detailed elsewhere. $^{22}$ The first appointment occurred at an oncampus laboratory. On that day, participants were to have abstained from cannabis before their appointment (confirmed through self-report). After informed consent, participants completed breathalyzer and urine drug tests to check for alcohol, sedatives, cocaine, opiates, or amphetamines in their system. Those failing either test or reporting cannabis use earlier that day were rescheduled; repeated failures resulted in termination. Females took a urine pregnancy test to ensure they were not pregnant. Participants then completed questionnaires asking about demographics, lifestyle, psychological factors, substance use, and medical history (see Supplementary Data). Next, participants completed a set of impairment assessments, including the DRUID app, to establish their baseline sober level of functioning. Finally, participants were randomly assigned to purchase one of two cannabis products at a local dispensary (The Farm; https://thefarmco.com/). Those who were primarily flower users $(n=16$ included in this study) were directed to purchase $3 \mathrm{~g}$ of their assigned flower product, containing either $16 \%$ or $24 \%$ THC; those who were primarily concentrate users ( $n=54$ included in this study) were directed to buy $1 \mathrm{~g}$ of their assigned concentrate, with either $70 \%$ or $90 \%$ THC. Consistent with the State of Colorado requirements, the THC potency of each study product was labeled after batch testing in an International Organization of Standards 17025-accredited laboratory. The cannabidiol levels of all products were below the laboratory's detection limit (i.e., were close to zero).

The second appointment took place in a mobile laboratory driven to participants' residence. Before their appointment, participants were to have abstained from alcohol for $24 \mathrm{~h}$ (verified through breathalyzer) and from cannabis that day (verified by self-report). Participants completed the same set of impairment assessments three times. After the first assessment (preuse time point), participants went into their homes to use their assigned cannabis ad libitum, and then returned immediately to the mobile laboratory to complete the assessments a second time (acute postuse time point). They remained in the mobile laboratory for $1 \mathrm{~h}$ and then completed the assessments for a third and final time ( $1 \mathrm{~h}$ postuse time point). Blood was also drawn at each time point to objectively measure the level of cannabinoids in participants' blood when they completed each assessment (see Supplementary Data for blood collection and assay methods).

Measures. Taking $\sim 2 \mathrm{~min}$, the DRUID mobile app assesses psychomotor and cognitive impairment by having users complete four tasks that call upon several capabilities potentially affected by cannabis: eyes-open balance, reaction time, and performance on a psychomotor vigilance task, ${ }^{25}$ hand-eye coordination, ${ }^{26}$ attention to motion stimuli, ${ }^{27}$ and time estimation. ${ }^{28}$ The tasks are as follows (see Supplementary Data for additional details): Task 1 -reaction time, decision making, and divided attention; Task 2 -reaction time, time estimation, and divided attention; Task 3-motion tracking and divided attention; Task 4-eyes-open balance.

DRUID integrates task-level intermediate variables by using a proprietary algorithm to produce a total impairment score, our primary outcome measure. Impairment scores range from 0 to 100 . The present analysis includes task-level data as exploratory outcomes. Note that total scores might indicate statistically significant increases in impairment even when one or more tasklevel measures do not show significant decrements.

\section{Washington study}

\section{Procedure}

The research protocol was approved by the New England IRB, Needham, MA (https://neirb.com/). For this study, frequent cannabis users $(n=39)$ were recruited from Seattle and Leavenworth, WA, using informal networks 
accessed by The Cannabis Alliance, a Washington-based group of state-licensed businesses, and SMJ Consulting, part of the Advanced Integrative Medical Science (AIMS) Institute. To be included, participants had to be at least 21 years and report daily or near daily cannabis use. Enrolled participants agreed to avoid alcohol and cannabis use for at least $8 \mathrm{~h}$ before their scheduled session (verified through self-report). On site, females completed a urine pregnancy test, all of which were negative.

Participants completed a single study session lasting $3 \mathrm{~h}$, conducted at either the AIMS Institute or a private home in Leavenworth. In the first $30 \mathrm{~min}$, participants practiced the DRUID app three to four times and then established their sober baseline performance. They were then asked to consume the amount and type of cannabis they typically used, consisting of either a concentrate ( $n=2$ participants) or flower cannabis prerolled into $0.5 \mathrm{~g}$ "joints" $(n=37$, with no participant using $<1 \mathrm{~g})$. The cannabis products varied between $17 \%$ and $21 \%$ THC. Participants were given $20 \mathrm{~min}$ to consume their cannabis. Next, 30 min after initiating consumption, they used DRUID to assess their impairment and then again every $30 \mathrm{~min}$ through $2.5 \mathrm{~h}$ postinitiation.

\section{Data analysis}

Statistical analyses were conducted in SAS (version 9.4), using Proc Mixed for both the Colorado and Washington study analyses. Random coefficient regression models with random intercepts were used to assess withinsubject changes in total impairment scores and tasklevel performance across the study time points. The total impairment score is the primary outcome variable generated by the DRUID app. Individual task-level data are thus considered exploratory, as prior research has not been conducted to validate DRUID individual task-level performance scores. Proc Mixed utilizes modern maximum likelihood-based approaches to handle missing data, thereby allowing utilization of all possible data points in the analyses. ${ }^{29}$ All models included age, gender, and cannabis type (cannabis type was considered only for the Colorado study only, which had representative number of both flower and concentrate users) as covariates, and both linear and quadratic effects of time on the relevant total impairment or task score. Our prior study tested the effects of flower and concentrate products on subjective and objective impairment measures, and no effects emerged, ${ }^{22}$ thus concentration of THC was not included as a covariate in this analyses. A similar analysis strategy for the Washington sample focused only on the total impair- ment score. The Washington models also included a group variable indicating whether participants were recruited from Seattle or Leavenworth, WA.

\section{Results}

\section{Colorado study}

Participant characteristics. Of the 76 participants enrolled in the study, 6 were excluded from the data analysis because their blood cannabinoid levels at the first postcannabis-use time point were $<20 \mathrm{ng} / \mathrm{mL}$, indicating they had not followed the cannabis use instructions. The remaining 70 participants (mean age $=28.93$ years, $44 \%$ female) included 16 flower and 54 concentrate users. Note that for DRUID performance variables, $n=66$ for the immediate postuse time point, and $n=65$ for the $1 \mathrm{~h}$ postuse time point, as several individuals did not complete all assessment time points. There were few baseline differences between the flower and concentrate groups (Table 1). As expected, each group reported more frequent use of the cannabis type they had chosen for the study. The concentrate group had significantly higher baseline levels of the THC metabolite THC$\mathrm{COOH}$, whereas the flower group reported using significantly more of their product ad libitum during the second appointment.

Changes in DRUID total impairment scores. The DRUID total impairment score showed a significant quadratic effect of time $(\beta=-0.935, S E=0.204, p<0.001)$ and a marginal linear effect $(\beta=0.344, S E=0.1891$, $p=0.071)$. As shown in Figure 1, the participants experienced peak impairment at the acute postuse time point, with a mean DRUID score of 50.6. There was also a significant main effect of age on total impairment score $(\beta=0.395, S E=0.062, p<0.001)$. There were no significant effects for cannabis type.

Changes in task-level results. For Task 2 (reaction time, time estimation, and divided attention), there were significant quadratic $(\beta=-0.0022, S E=0.0007$, $p=0.0014)$ and linear effects $(\beta=0.0038, S E=0.0015$, $p=0.0152$ ) of time on average error distance (see Supplementary Data) and quadratic effects $(\beta=-0.0287$, $S E=0.0087, p=0.0013)$ of time on average reaction time. For Task 4 (eyes-open balance), there was a significant quadratic effect of time on total movement $(\beta=-3.3383, S E=0.8166, p<0.0001)$, right leg movement $(\beta=-1.6196, S E=0.4017, p<0.0001)$, and left leg movement $(\beta=-1.6767, S E=0.4397, p=0.0002)$. There were also significant effects of age and gender on total 
Table 1. Participant Characteristics Across Colorado Study Flower and Concentrate Users (CO)

\begin{tabular}{|c|c|c|c|}
\hline Demographics & $\begin{array}{c}\text { Overall CO sample } \\
\qquad \begin{array}{c}n=70 \\
\mathrm{M}(S D)\end{array}\end{array}$ & $\begin{array}{l}\text { Flower users } \\
\begin{array}{c}n=16 \\
M(S D)\end{array}\end{array}$ & $\begin{array}{l}\text { Concentrate } \\
\text { users } n=54 \\
M(S D)\end{array}$ \\
\hline Age & $28.93(11.9)$ & $14.10(3.5)$ & $11.26(1.5)$ \\
\hline Gender (female), $n$ (\%) & $31(44 \%)$ & $5(31 \%)$ & $26(48 \%)$ \\
\hline Marital status (married), $n$ (\%) & $9(13 \%)$ & $2(13 \%)$ & $7(13 \%)$ \\
\hline Education (bachelors or higher), $n(\%)$ & $34(49 \%)$ & $10(63 \%)$ & $24(44 \%)$ \\
\hline Race (white), $n(\%)$ & $53(76 \%)$ & $11(69 \%)$ & $42(78 \%)$ \\
\hline \multicolumn{4}{|l|}{ Cannabis use history and use measures } \\
\hline Age of onset of regular cannabis use & $18.22(5.6)$ & $19.69(2.7)$ & $17.78(6.1)$ \\
\hline Days of concentrate use $\mathrm{a}^{\mathrm{a}}$ & $12.97(11.9)$ & $2.94(6.8)^{* * *}$ & $15.94(11.4)^{* * * *}$ \\
\hline Days of flower use $\mathrm{e}^{\mathrm{a}}$ & $17.46(10.5)$ & $23.50(6.5)^{* * *}$ & $15.67(10.8)^{* * * *}$ \\
\hline MDS & $3.19(2.2)$ & $3.44(2.4)$ & $3.11(2.2)$ \\
\hline Baseline plasma THC (ng/mL) & $8.20(9.8)$ & $5.62(9.1)$ & $8.96(9.9)$ \\
\hline Baseline plasma 11-OH-THC (ng/mL) & $3.03(4.8)$ & $1.90(3.1)$ & $3.37(5.1)$ \\
\hline Baseline plasma THC-COOH (ng/mL) & $83.56(86.2)$ & $49.09(78.3)$ & $93.77(86.4)$ \\
\hline \multicolumn{4}{|l|}{ Cannabis use during ad libitum administration } \\
\hline Grams used during experimental appointment ${ }^{b}$ & $0.14(0.2)$ & $0.27(0.2)^{*}$ & $0.11(0.1)^{*}$ \\
\hline Time away from van (minutes) ${ }^{c}$ & $13.34(7.1)$ & $12.10(3.8)$ & $13.57(7.5)$ \\
\hline \multicolumn{4}{|l|}{ Other substance use and psychological factors } \\
\hline AUDIT total & $6.37(4.6)$ & $5.25(2.5)$ & $6.70(5.0)$ \\
\hline Depression (BDI total) & $6.21(6.0)$ & $4.31(4.1)$ & $6.78(6.4)$ \\
\hline Anxiety (BAI total) & $5.64(5.9)$ & $4.69(5.2)$ & $5.93(6.1)$ \\
\hline
\end{tabular}

a Using a 30-day timeline follow back.

${ }^{b}$ Participants brought our scale into their home to measure the amount of study cannabis used during the experimental appointment.

'The time participants spent inside their homes during the cannabis administration was recorded, but this procedure was implemented after the first six subjects had been run. Thus, $n=64$ for this variable.

${ }^{* \prime}$ s indicate a significant difference emerged in the tests comparing the two user groups (flower vs. concentrate). ${ }^{*} p<0.05,{ }^{* * *} p \leq 0.001$.

AUDIT, Alcohol Use Disorders Indentification Test; BAI, Beck Anxiety Inventory; BDI, Beck Depression Inventory-Il; MDS, Marijuana Dependence Scale Score.

movement (age $\beta=1.4657, S E=0.2562, p<0.0001$; gender $\beta=12.6653, S E=6.1043, p=0.0419$ ), right leg movement (age $\beta=0.9435, S E=0.1580, p<0.0001$; gender $\beta=7.7344, S E=3.7545, p=0.0433$ ), and left leg movement (age $\beta=0.6298, S E=0.1272, p<0.0001$; gender $\beta=8.1090, S E=3.0315, p=0.0094)$. Models indicated greater impairment in older male participants.

There were no significant changes over time for Task 1 (reaction time, decision making, and divided attention); a significant effect of age emerged for average reaction time $(\beta=0.0036, S E=0.0010, p=0.0011)$, where older participants were more impaired. Likewise, there were no significant changes over time for Task 3 (motion tracking and divided attention), but a significant effect of gender emerged for the percentage of time the participants' finger was not touching the moving target $(\beta=-9.6051, S E=1.9332, p<0.0001)$, such that female participants were more impaired.

Washington study

Participant characteristics. Thirty-nine participants (mean age $=39.5$ years; $35.9 \%$ female) were included in the analysis (Table 2).

Changes in DRUID total impairment scores. The DRUID total impairment score showed a signifi- cant quadratic effect of time $(\beta=3.0299, S E=1.3085$, $p<0.01)$. There were also significant effects of age, gender, and group (Seattle vs. Leavenworth) on total impairment (age $\beta=0.5374, S E=0.1088, p<0.0001$; gender $\beta=4.2503$, $S E=2.0465, p<0.05$, group $\beta=6.3268, S E=1.9876$, $p<0.01)$. In this sample, older male individuals were more impaired.

Figure 1 shows the mean DRUID total impairment scores for the Colorado and Washington participants. The average peak DRUID score for Washington participants was $50.7(S D=12.13)$. Colorado participants experienced peak impairment at the first postuse time point, with a mean score of $50.6(S D=11.0)$. Beginning at $2 \mathrm{~h}$ postinitiation ( $1.5 \mathrm{~h}$ after finishing consumption), there was no longer a significant difference between participants' preconsumption and postconsumption scores in the Washington sample $\left(t_{(30)}=1.38, p>0.15\right)$.

\section{Discussion}

Recent changes to cannabis policy and increasing cannabis use have raised public safety concerns, especially regarding cannabis-related psychomotor impairment that may impact driving, yet there is no valid and reliable assessment method for identifying cannabis userelated acute impairment. Furthermore, any behavioral 


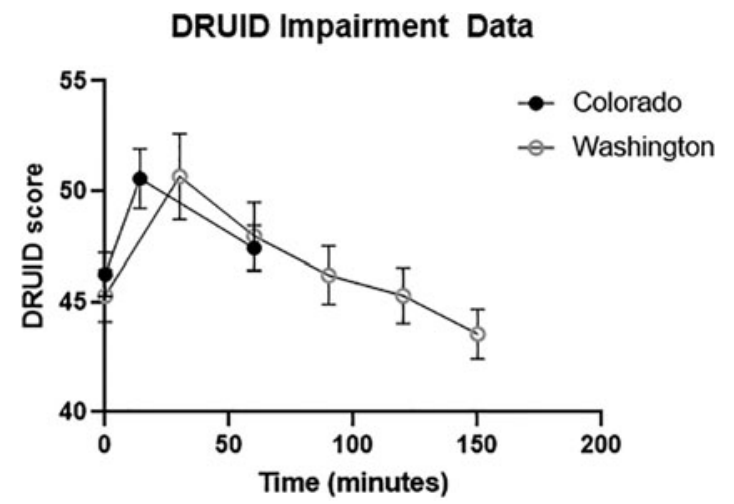

FIG. 1. DRUID ${ }^{\circledR}$ total impairment scores after acute cannabis ingestion across Colorado and Washington samples. The $\mathrm{Y}$-axis represents DRUID total impairment score. On the $\mathrm{X}$-axis, " 0 " represents the preuse time point. The second time point for the Colorado study was $\sim 14$ min later, as individuals spent an average of $14 \mathrm{~min}$ in their homes consuming their cannabis product, and the postuse measurement was taken immediately when they returned to the mobile laboratory. The second time point for the Washington sample took place $\sim 30 \mathrm{~min}$ after subjects initiated consumption. Subjects took an average of $20 \mathrm{~min}$ to consume their product. Both studies took a third measurement $60 \mathrm{~min}$ after the baseline measurement, and the Washington sample took three more measurements every $30 \mathrm{~min}$.

metric of cannabis impairment would need to account for the tolerance to the effects of cannabis that occurs among frequent users, and even then, interpretations of individual performance would be limited due to the fact that individuals likely have dif-

Table 2. Basic Participant Characteristics, Washington Sample

\begin{tabular}{|c|c|c|c|}
\hline & $\begin{array}{c}\text { Overall } \\
n=39\end{array}$ & $\begin{array}{c}\text { Seattle } \\
n=19\end{array}$ & $\begin{array}{l}\text { Leavenworth } \\
\qquad n=20\end{array}$ \\
\hline & $M(S D)$ & $M(S D)$ & $M(S D)$ \\
\hline \multicolumn{4}{|l|}{ Demographics } \\
\hline Age & $39.54(8.80)$ & $37.63(9.77)$ & $41.35(7.57)$ \\
\hline Gender (female), $n$ (\%) & $14(35.9 \%)$ & $10(52.6 \%)$ & $4(20 \%)^{*}$ \\
\hline \multicolumn{4}{|l|}{ Cannabis use history } \\
\hline $\begin{array}{l}\text { Age of onset of } \\
\text { frequent cannabis } \\
\text { use }\end{array}$ & $20.82(7.68)$ & $18.53(5.34)$ & $23.00(8.99)$ \\
\hline
\end{tabular}

fering levels of baseline (i.e., nonintoxicated) performance on tasks that measure psychomotor and neurocognitive abilities such as balance and reaction time. Taking these practical limitations into account, we used the DRUID app to examine the magnitude and time course of impairment experienced by frequent users after naturalistic cannabis use.

Both the Colorado and Washington studies demonstrated that frequent cannabis users consuming products consistent with their typical use patterns had significantly increased impairment after consuming cannabis with a high THC concentration. In addition, scores were similar between the two samples after using cannabis (50.7 for the WA samples and 50.6 for the CO samples). The Colorado study explicitly tested whether impairment differed based on the type of cannabis used (e.g., use of flower (16-24\% THC) versus concentrate (70-90\%) and found that impairment was similar across groups. The most notable finding was the fact that in both studies, impairment peaked shortly after acute use and decreased over the next hour. Both studies also demonstrated consistently higher impairment among older participants. Gender differences, however, were less clear; in the Colorado sample, males demonstrated greater impairment on the balance task, and females demonstrated greater impairment on the motion tracking task. Total impairment in the Washington sample was greater in males, whereas no significant gender differences emerged for total impairment in the Colorado sample. Thus, additional research is needed to better understand the potential effects of gender on various aspects of cannabis-related impairment. Overall external validity of these findings is enhanced by the fact that there was variability in the amount of cannabis consumed, type of cannabis (i.e., flower or concentrate), and method of administration.

It is worth noting that the preuse DRUID scores in the present samples (45 in the WA sample and 46 in the CO sample) were similar to the average preintoxication DRUID score of 44 obtained in a prior study of 47 regular alcohol users. ${ }^{30}$ This suggests that on average, substance using populations score in a similar range on the DRUID preintoxication. Based on data collected with alcohol-impaired volunteers, ${ }^{30}$ cannabis-impaired volunteers, ${ }^{24}$ and $>12,000$ additional users under various testing conditions, the makers of DRUID suggest that scores can be interpreted as follows: $<44=$ no impairment; $44-48=$ mild impairment; $48-52=$ moderate 
impairment; 52-57= high impairment; 57-62=very high impairment; and $>62=$ severe impairment. Further research is needed to explore whether preintoxication scores observed in the present study are comparable with other populations of both substance users and nonusers.

Specific task-level effects emerged for simple postural stability and reaction time in the context of a complex divided attention task, such that balance and reaction time were impaired immediately postuse, but improved $1 \mathrm{~h}$ later. This significant effect of cannabis, regardless of type, on balance function is consistent with prior work indicating that THC acutely impairs balance function, including increasing body sway ${ }^{31-33}$ and postural tracking. ${ }^{34}$ Furthermore, the significant quadratic effect on the DRUID app's balance test is consistent with our prior study of flower cannabis and concentrate users that used a different method to assess balance function. ${ }^{22}$ We also detected slower reaction times during a complex divided-attention DRUID task that called for participants to estimate when $30 \mathrm{sec}$ had elapsed, a finding consistent with prior studies that showed THC ingestion is associated with slower response times when performing several types of tasks (e.g., simple reaction time, selective attention, sustained attention, and divided attention) $)^{35}$ and during driving simulations. ${ }^{36}$ We did not observe any effects for reaction time during a motion-tracking task. We also failed to observe any significant effects on time estimation itself, although reaction times were slower during the time estimation task. Research on how cannabis affects time estimation has been inconclusive, ${ }^{37}$ although impaired performance after acute cannabis use may be more likely among infrequent users than frequent users. ${ }^{28}$

Taken together, these results suggest that when using cannabis ad libitum, frequent users experienced balance and reaction time impairment immediately after using cannabis, which then decreases substantially within $1 \mathrm{~h}$ postuse. This finding stands in contrast to a neuroimaging study that found that frequent cannabis users may experience deficits in brain activation in motor control regions that may persist well beyond acute intoxication. ${ }^{16}$ It is possible that individuals in this study would have shown some form of persistent psychomotor or neurocognitive impairment that were not included in the DRUID battery when tested after an even longer postuse period. Overall, regarding the individual task-level results, it is important to note that these tests were considered exploratory and are primarily intended to facilitate future research and inform further studies in this area. In addition, our lack of follow-up data or a placebo group precludes our ability to definitively determine whether these users experience any persistent impairments related to their cannabis use.

At present, DRUID has several limitations that preclude its use as a law enforcement tool. It would not be possible for law enforcement to compare individuals' roadside performance with their nonintoxicated performance, and research has not yet linked specific scores on the DRUID to objective driving impairment. Furthermore, the differences observed in this study do not allow us to draw conclusions or make inferences about whether an individual has used cannabis based on their DRUID performance. With these limitations in mind, the present studies suggest that the underlying psychomotor functions measured by DRUID are sensitive to impairment that occurs immediately after cannabis consumption, as well as to the performance rebound that appears to occur $1 \mathrm{~h}$ after use. In summary, there remains a need for a reliable tool to assess cannabisrelated impairment for law enforcement purposes. ${ }^{3,4,6}$

\section{Limitations and future directions}

Although the general impairment patterns described in prior literature were supported by these two studies, future research should test the DRUID app in a broader range of circumstances and among individuals with varying demographic characteristics to identify the patterns of DRUID results, both short and longer term, associated with different user profiles. One important limitation is the fact that this study excluded cannabis users who were also heavy tobacco and/or alcohol users. Given that polydrug use between cannabis and both alcohol and tobacco is common, ${ }^{38,39}$ future studies should include users of all three substances. The present analyses also lacked a placebo control condition, which should be included in future study. The lack of a placebo control condition in this study means that we cannot exclude drug expectancy effects as an explanation of our findings. In addition, as age and gender were significant covariates across the majority of the effects reported here, future studies should test age and gender as moderators of the acute effects of cannabis on psychomotor performance. It would also be useful to incorporate data regarding subjects' typical driving patterns (e.g., how often they operate a motor vehicle in general and how often they drive after using cannabis). This type of detailed data was not collected in this study; however, future studies that collect 
this information may be able to provide a useful realworld context for framing interpretations of DRUID or other motor performance findings. Finally, DRUID impairment has not yet been directly linked with driving simulator or other driving performance metrics, which is an important direction for future study.

\section{Conclusions}

This study demonstrated that regular cannabis users show a decrease in performance on the DRUID app immediately after cannabis ingestion, but appear to "recover" almost back to baseline levels $1 \mathrm{~h}$ postuse. This finding is notable, as it underscores the fact that even regular cannabis users are impaired in specific psychomotor domains after using cannabis, and law enforcement should have a method for measuring that impairment in drivers. At present, there is a great need for a wellsupported brief and mobile method for determining acute cannabis-related impairment, but no such tool exists. Considerable further validation and development are needed to determine whether a testing battery like DRUID may have individual or other applications as a driving impairment test.

\section{Author Disclosure Statement}

Dr. DeJong is a shareholder with Impairment Science, Inc. Dr. Milburn is the founder and chief science officer for Impairment Science, Inc.

\section{Funding Information}

Funding for this study was provided by grants from the National Institutes of Health (Grant No. DA039707) and Colorado Department of Public Health and Environment grant (Grant No. 96947 to L.C.B.) as well as from the National Institutes of Health (NIDA) SBIR Grant No. R44DA046272-01A1 to M.A.M./Impairment Science, Inc.

\section{Supplementary Material}

Supplementary Data

\section{References}

1. Mauro CM, Newswanger $P$, Santaella-Tenorio J, et al. Impact of medical marijuana laws on state-level marijuana use by age and gender, 20042013. Prev Sci. 2019;20:205-214.

2. Kelley-Baker T, Bearning A, Ramirez A, et al. 2013-2014 National Roadside Study of alcohol and drug use by drivers: drug results (No. DOT HS 812 411). National Highway Traffic Safety Administration: Washington, DC, USA, 2017.

3. Bosker WM, Kuypers KPC, Theunissen EL, et al. Medicinal $\triangle 9$ tetrahydrocannabinol (dronabinol) impairs on-the-road driving performance of occasional and heavy cannabis users but is not detected in Standard Field Sobriety Tests. Addiction. 2012;107:1837-1844.
4. Papafotiou K, Carter JD, Stough C. An evaluation of the sensitivity of the Standardised Field Sobriety Tests (SFSTs) to detect impairment due to marijuana intoxication. Psychopharmacology (Berl). 2005;180:107-114.

5. Logan BK, Kacinko SL, Beirness DJ. An evaluation of data from drivers arrested for driving under the influence in relation to per se limits for cannabis. AAA Foundation for Traffic Safety, Washington DC, USA, 2016.

6. Capler R, Bilsker D, Van Pelt K, et al. Cannabis use and driving: evidence review. Canadian Drug Policy Coalition (CDPC), Simon Fraser University: Vancouver, BC, 2017.

7. Compton R. Marijuana-impaired driving a report to congress (No. DOT HS 812 440). National Highway Traffic Safety Administration: Washington DC, USA, 2017.

8. Desrosiers NA, Ramaekers JG, Chauchard E, et al. Smoked cannabis' psychomotor and neurocognitive effects in occasional and frequent smokers. J Anal Toxicol. 2015;39:251-261.

9. Hartman RL, Brown TL, Milavetz G, et al. Cannabis effects on driving lateral control with and without alcohol. Drug Alcohol Depend. 2015;154:25-37.

10. Hartman RL, Huestis MA. Cannabis effects on driving skills. Clin Chem. 2013;59:478-492.

11. Karschner EL, Swortwood MJ, Hirvonen J, et al. Extended plasma cannabinoid excretion in chronic frequent cannabis smokers during sustained abstinence and correlation with psychomotor performance. Drug Test Anal. 2016;8:682-689.

12. Bolbecker AR, Apthorp D, Martin AS, et al. Disturbances of postural sway components in cannabis users. Drug Alcohol Depend. 2018;190:54-61.

13. Metrik J, Kahler CW, Reynolds B, et al. Balanced placebo design with marijuana: pharmacological and expectancy effects on impulsivity and risk taking. Psychopharmacology (Berl). 2012;223:489-499.

14. van Wel JHP, Kuypers KPC, Theunissen EL, et al. Single doses of THC and cocaine decrease proficiency of impulse control in heavy cannabis users. Br J Pharmacol. 2013;170:1410-1420.

15. Crean RD, Crane NA, Mason BJ. An evidence based review of acute and long-term effects of cannabis use on executive cognitive functions. J Addict Med. 2011;5:1-8.

16. Pillay SS, Rogowska J, Kanayama G, et al. Cannabis and motor function: fMRI changes following 28 days of discontinuation. Exp Clin Psychopharmacol. 2008;16:22-32.

17. Claudet I, Mouvier S, Labadie M, et al. Unintentional cannabis intoxication in toddlers. Pediatrics. 2017;140:e20170017.

18. Mehmedic Z, Chandra S, Slade D, et al. Potency trends of $\triangle 9-T H C$ and other cannabinoids in confiscated cannabis preparations from 1993 to 2008. J Forensic Sci. 2010;55:1209-1217.

19. Orens $A$, Light $M$, Lewandowski $B$, et al. Market size and demand for marijuana in Colorado: 2017 market update prepared for the Colorado Department of Revenue. Marijuana Policy Group: Boulder, CO, 2017.

20. Johnson RM, Brooks-Russell A, Ma M, et al. Usual modes of marijuana consumption among high school students in Colorado. J Stud Alcohol Drugs. 2016;77:580-588.

21. Bidwell LC, Mueller R, YorkWilliams SL, et al. A novel observational method for assessing acute responses to cannabis: preliminary validation using legal market strains. Cannabis Cannabinoid Res. 2018;3:35-44.

22. Bidwell LC, Ellingson JM, Karoly HC, et al. Association of Naturalistic Administration of Cannabis Flower and Concentrates With Intoxication and Impairment. JAMA Psychiatry. 2020;77:787-796.

23. Richman JE. An investigation of the Druid ${ }^{\circledR}$ smartphone/tablet app as a rapid screening assessment for cognitive and psychomotor impairment associated with alcohol intoxication. 2019;5:31-42.

24. Spindle T. "Cannabis drug testing and measurement of impairment." National Safety Council meeting on Cannabis Use in the Workplace ("Cannabis, its Complicated"), 6/26/2019. Sponsor: Jane Terry, Chicago, IL, 2019.

25. Jongen S, Vuurman E, Ramaekers J, et al. Alcohol calibration of tests measuring skills related to car driving. Psychopharmacology (Berl). 2014; 231:2435-2447.

26. Moskowitz H. Marihuana and driving. Accid Anal Prev. 1985;17:323-345.

27. Mikulskaya E, Martin FH. Contrast sensitivity and motion discrimination in cannabis users. Psychopharmacology (Berl). 2018;235:2459-2469.

28. Sewell RA, Schnakenberg A, Elander J, et al. Acute effects of THC on time perception in frequent and infrequent cannabis users. Psychopharmacology (Berl). 2013;226:401-413.

29. Schafer JL, Graham JW. Missing data: our view of the state of the art. Psychol Methods. 2002;7:147-177. 
30. Richman JE, May S. An investigation of the DRUID ${ }^{\circledR}$ smartphone/tablet app as a rapid screening assessment for cognitive and psychomotor impairment associated with alcohol intoxication. Vis Dev Rehabil. 2019;5: 31-42.

31. Zuurman L, Roy C, Schoemaker RC, et al. Effect of intrapulmonary tetrahydrocannabinol administration in humans. J Psychopharmacol. 2008;22: 707-716.

32. Klumpers LE, Beumer TL, van Hasselt JGC, et al. Novel $\Delta 9$ tetrahydrocannabinol formulation Namisol ${ }^{\circledR}$ has beneficial pharmacokinetics and promising pharmacodynamic effects. Br J Clin Pharmacol. 2012;74:42-53.

33. Liguori A, Gatto CP, Jarrett DB. Separate and combined effects of marijuana and alcohol on mood, equilibrium and simulated driving. Psychopharmacology (Berl). 2002;163:399-405.

34. Greenberg HS, Werness SAS, Pugh JE, et al. Short-term effects of smoking marijuana on balance in patients with multiple sclerosis and normal volunteers. Clin Pharmacol Ther. 1994;55:324-328.

35. Hunault CC, Mensinga TT, Böcker KBE, et al. Cognitive and psychomotor effects in males after smoking a combination of tobacco and cannabis containing up to $69 \mathrm{mg}$ delta-9-tetrahydrocannabinol (THC). Psychopharmacology (Berl). 2009;204:85-94.

36. Lenné MG, Dietze PM, Triggs TJ, et al. The effects of cannabis and alcohol on simulated arterial driving: influences of driving experience and task demand. Accid Anal Prev. 2010;42:859-866.

37. Atakan Z, Morrison P, Bossong MG, et al. The effect of cannabis on perception of time: a critical review. Curr Pharm Des. 2012;18:4915-4922.
38. Peters EN, Budney AJ, Carroll KM. Clinical correlates of co-occurring cannabis and tobacco use: a systematic review. Addiction. 2012;107: 1404-1417.

39. Subbaraman MS, Kerr WC. Simultaneous versus concurrent use of alcohol and cannabis in the National Alcohol Survey. Alcohol Clin Exp Res. 2015; 39:872-879.

Cite this article as: Karoly $\mathrm{HC}$, Milburn MA, Brooks-Russell $A$, Brown $M$, Streufert J, Bryan AD, Lovrich NP, DeJong W, Cinnamon Bidwell L (2022) Effects of high-potency cannabis on psychomotor performance in frequent cannabis users, Cannabis and Cannabinoid Research 7:1, 107-115, DOI: 10.1089/can.2020.0048.

Abbreviations Used
$\mathrm{AIMS}=$ Advanced Integrative Medical Science
$\mathrm{AUDIT}=$ Alcohol Use Disorders Identification Test
$\mathrm{BAI}=$ Beck Anxiety Inventory
$\mathrm{BDI}=$ Beck Depression Inventory-II
$\mathrm{MDS}=$ Marijuana Dependence Scale Score
$\mathrm{THC}=$ delta-9-tetrahydrocannabinol

